\title{
HABILIDADE EMPÁTICA E SEU APRENDIZADO EM GRADUANDOS DE ENFERMAGEM
}

Leticia Serra Rondon ${ }^{1}$

Isabel Cristina Kowal OIm Cunha ${ }^{1}$

Francisco Rosemiro Guimarães Ximenes Neto ${ }^{2}$ https://orcid.org/0000-0001-9785-2709

https://orcid.org/0000-0001-6374-5665

https://orcid.org/0000-0002-7905-9990

Objetivo: Buscar evidências disponiveis na literatura sobre abordagem da empatia/habilidade empática em estudantes de Enfermagem. Método: Revisão integrativa da literatura nas bases de dados PubMed, LILACS e SciELO, resultando em 11 artigos. Resultados: Os estudos foram publicados entre 2013 e 2019, com três artigos em revistas com Qualis Capes, sendo três em revistas internacionais. O país com maior número de estudos na temática foi o Brasil, com sete, seguido de Portugal, México, Colômbia e Cuba com um estudo cada. A maior parte dos autores eram doutores (seis). O delineamento dos estudos, eram tanto qualitativos, quanto quantitativos (cinco) e a maior parte tinha nível de evidência IV. A análise de conteúdo identificou as categorias temáticas: Percepção do estudante de Enfermagem acerca do cuidado e empatia; Reflexão sobre empatia e a Enfermagem; e Habilidade empática e suas competências. Conclusão: A empatia é vista como capacidade essencial na formação de estudantes de Enfermagem e está diretamente relacionada ao cuidado humanizado e pessoal por parte da equipe de saúde. Esta habilidade deve fazer parte da matriz curricular em todos os cursos da saúde para fortalecer a afetividade durante o processo de cuidar. Descritores: Empatia; Educação em Enfermagem; Cuidados de Enfermagem; Estudantes; Estudantes de Enfermagem.

\section{EMPATHIC SKILLAND ITS LEARNING IN NURSING GRADUATES}

Objective: To search for available evidence in the literature on how empathy / empathic ability in nursing students is approached. Method: Integrative literature review, for selection, three PubMed, LILACS and SciELO databases resulted of 11 articles. Results: Most studies were published in 2013 and 2019, with 27.3\% in journals with classification in Brazil on Qualis Capes Bl and $18.2 \%$ in $\mathrm{Al}, 3$ was in international journals, and $45.5 \%$ in both English and Portuguese. The country with the largest number of studies on the subject was Brazil, with seven (64\%), followed by Portugal, Mexico, Colombia and Cuba with one (9\%) study each. Most of the authors were doctors (55.3\%). The approuches of the studies were both quali and quantitative (45.4\%) and most had level of evidence IV. The content analysis identified the thematic categories: (1) the nursing student's perception of care and empathy; (2) reflection on empathy and nursing; and (3) empathic ability and competencies. Conclusion: Empathy is seen as an essential capacity in the training of nursing students and is directly related to humanized and more personal care by the health team. This skill must be part of the teaching grid in all areas of health and all they benefit from their learning.

Descriptors: Empathy; Education, Nursing; Nursing Care; Students; Students, Nursing.

\section{HABILIDAD EMPÁTICA Y SU APRENDIZAJE EN GRADUADOS EN ENFERMERÍA}

Objectivo: Buscar evidencia disponible en la literatura sobre cómo se aborda la empatía/habilidad empática en estudiantes de enfermería. Método: Revisión bibliográfica integral em las bases de datos PubMed, LILACS y SciELO resultando en 11 artículos. Resultados: La mayoría de los estudios se publicaron en 2013 y 2019, con un 27,3\% en revistas con classificação en Brasil Qualis Capes $\mathrm{Bl}$ y un 18,2\% en Al, 3 en revistas internacionales y un 45,5\% en inglés y portugués. El país con el mayor número de estudios sobre el tema fue Brasil, con siete (64\%), seguido de Portugal, México, Colombia y Cuba con un (9\%) estudio cada uno. La mayoría de los autores eran médicos (55,3\%). Los diseños de los estudios fueron tanto cualitativos como cuantitativos $(45,4 \%)$ y la mayoría tenía un nivel de evidencia IV. El análisis de contenido identificó las categorías temáticas: (1) la percepción de atención y empatía del estudiante de enfermería; (2) reflexión sobre empatía y enfermería; y (3) capacidad y competencias empáticas. Conclusion: La empatía es vista como una capacidad esencial en la formación de estudiantes de enfermería y está directamente relacionada con la atención humanizada y más personal del equipo de salud. Esta habilidad debe ser parte de la red de enseñanza en todas las áreas de la salud y todos se benefician de su aprendizaje. Descriptores: Empatía; Educación en Enfermería; Atención de Enfermeira; Estudiantes; Estudiantes de Enfermeira.

IUniversidade Federal de São Paulo (UNIFESP), SP.

Universidade Estadual Vale do Acaraú (UVA), Sobral, CE.

Autor Correspondente: Isabel Cristina Kowal Olm Cunha Email: isabelcunha@unifesp.br

Recebido:10/4/2020 Aceito: 10/7/2020 


\section{INTRODUÇÃO}

A Enfermagem moderna estabelecida a partir dos preceitos teóricos e epistemológicos de Florence Nightingale, com foco na técnica e na ciência, definiu uma nova práxis de cuidado com base no ambiente, na pessoa e nas condições de saúde. Para tal, os profissionais deveriam basear suas práticas em princípios e normas, que em conjunto com a formação acabaram por definir a Enfermagem enquanto profissão $0^{1,2}$, com reconhecimento social, legal e institucional em diversos países.

A arte do cuidado ganha novas definições e a Enfermagem como profissão deixa de ser apenas a repetição de atividades e o cumprimento de tarefas, caracterizando-se por um trabalho pluralizado e complexo, exigindo do enfermeiro conhecimentos diversos e um grande autoconhecimento, maturidade e estabilidade emocional, com ética e respeito às crenças, valores e culturas, para que a empatia com seu cliente ocorra ${ }^{3}$ a partir de um encontro afetivo, carregado de subjetividade, oportunizando uma dialética entre cuidador e sujeito de cuidado.

O elemento cognitivo da empatia pode-se caracterizar pela capacidade de deduzir os sentimentos e pensamentos de outro, sem a necessidade de experimentá-los. Esta é uma perspectiva que envolve sentimentos neutros e imparciais do sujeito que está exercendo a empatia e tem sido nomeado de tomada de perspectiva. Já na empatia afetiva, é possivel vivenciar sentimentos de compaixão, preocupação e simpatia com o bem-estar do outro. Este componente não tem implicância necessária em experimentar os mesmos sentimentos do outro, mas sim entender o que este está sentindo ou sentir uma espécie de afeto, que está relacionado à situação em que o outro se encontra mais do que para a situação em si ${ }^{4}$.

O conceito da empatia na área da saúde pode ter diversas variáveis, emocionais e cognitivas. Pode-se compreender empatia como a capacidade de ouvir, compreender e compadecer-se ao colocar-se no lugar do outro. Após o reconhecimento do sentimento do outro, é necessário auxiliá-lo. Este tipo de empatia é denominado de comportamental e pode incluir comportamentos verbais ou não verbais. A empatia comportamental é fundamental para que o cliente se sinta completamente compreendido durante o processo de cuidar, favorecendo o relacionamento entre este e o enfermeiro ${ }^{5}$.

Destarte, a formação do enfermeiro tem como finalidade o preparo do profissional para a realização de competências e habilidades voltadas para a atenção à saúde, tomada de decisões, comunicação, liderança, administração e gerenciamento e a educação permanente. Pois, a Enfer- magem não é apenas uma ciência focada na saúde numa perspectiva biológica, mas no humanismo ${ }^{6}$, o que exige um ensino baseado em competências socioemocionais, para a transformação social da profissão e das atitudes de cada profissional durante o processo saúde-doença-cuidado ${ }^{7}$.

A prática da Enfermagem tem efeitos muito importantes quando o profissional demonstra empatia, por esta influenciar fortemente a relação cliente-enfermeiro. Presume-se que esta relação terapêutica seja traduzida em comportamentos de melhor cuidado como atenção, escuta e sentimentos de ajuda $a^{5}$. Contribuindo para mais assertividade nos diversos e adversos momentos de cuidado que circundam desde o nascimento até as condições extremas de sofrimento, dor, doença e a morte.

Considerando a empatia uma habilidade essencial e que influencia diretamente nas relações e na subjetividade envolta ao processo de cuidar da Enfermagem, este estudo tem como objetivo buscar evidências disponiveis na literatura sobre como é abordada a empatia/habilidade empática com estudantes de Enfermagem.

\section{MÉTODO}

\section{Tipo de Estudo}

Trata-se de revisão integrativa, que permite incluir literatura teórica e baseada na experiência, além de estudos com diferentes desenhos metodológicos (qualitativos e quantitativos) $^{8}$.

\section{Etapas da revisão integrativa}

Para a realização da revisão foram permeadas seis etapas: a) identificação do tema e seleção da questão de pesquisa; b) estabelecimento dos critérios de inclusão/ exclusão (seleção da amostra); b) definição dos estudos pré-selecionados e selecionados; c) categorização dos estudos; d) análise e interpretação dos resultados; e e) apresentação da revisão/síntese do conhecimento?.

No que se refere a construção da questão de pesquisa da revisão integrativa, utilizou-se a estratégia PICOT (acrônimo para Patient, Intervention, Comparison, Outcomes e Time). A estratégia utilizada para esta construção permite a identificação de palavras-chave, as quais possibilitam a melhor localização de estudos relevantes nas bases de dados ${ }^{10}$.

No Quadro 1, estão dispostos os elementos utilizados para construir a pergunta de pesquisa e os descritores a partir da estratégia PICOT ${ }^{11}$. Destaca-se que, dependendo do método de revisão, não se emprega todos os elementos da estratégia PICOT. Nesta revisão integrativa, o terceiro elemento que é a comparação, não foi utilizada. 
Quadro 1 Descrição da estratégia Patient/population, Intervention or issue of interest, Comparison intervention or issue of interest, Outcomes e Time (PICOT) ${ }^{11}$.

\begin{tabular}{|c|c|c|}
\hline Acrônimo/ Definição & Proposta do estudo & Descritores DeCS/MeSH \\
\hline P: Patient/ population & $\begin{array}{l}\text { Estudantes de graduação } \\
\text { em enfermagem. }\end{array}$ & Estudantes de enfermagem; Nursing Students. \\
\hline I: Intervention/issue of interest & $\begin{array}{l}\text { Como é abordada a Empa- } \\
\text { tia/habilidade empática. }\end{array}$ & $\begin{array}{l}\text { Empatia; educação em enfermagem; cuidados de } \\
\text { enfermagem; estudantes; estudantes de enferma- } \\
\text { gem. } \\
\text { Empatia; Educación en Enfermería; Atención de } \\
\text { Enfermeira; Estudiantes; Estudiantes de Enfermeira. } \\
\text { Empathy; Education, Nursing; Nursing Care; Stu- } \\
\text { dents; Students, Nursing. }\end{array}$ \\
\hline O: outcomes - Resultados esperados & $\begin{array}{l}\text { Contribuição da habilidade } \\
\text { empática para a formação } \\
\text { do enfermeiro. }\end{array}$ & \\
\hline $\begin{array}{l}\text { T: time - Período de tempo } \\
\text { da intervenção }\end{array}$ & 2009 a 2019 & \\
\hline
\end{tabular}

Assim, a questão de pesquisa determinada foi: "Como é abordada a habilidade empática/empatia em estudantes de Enfermagem?".

Para a seleção dos artigos, foram utilizadas as bases de dados Literatura Latino-Americana e do Caribe em Ciências da Saúde (LILACS), Scientific Electronic Library Online (SciELO) e United States Nacional Library of Medicine/Nacional Institutes of Health (PubMed/MEDLINE). A coleta ocorreu entre os meses de janeiro e março de 2020.

Os descritores controlados selecionados nos Descritores em Ciência da Saúde (DeCS) da Biblioteca Virtual em Saúde (BVS) e MeSH Database (Medical Subject Headings) foram "Empatia”, “Cuidados de Enfermagem", “Enfermagem", "Estudantes" e "Estudantes de Enfermagem" (nos idiomas português, inglês e espanhol). A combinação dos descritores foi realizada por meio dos operadores booleanos "AND" (combinação restritiva) e "OR" (combinação aditiva), de diferentes formas para garantir busca ampla, cujos cruzamentos em todas as bases de dados foram: Empatia AND Cuidados de Enfermagem AND Estudantes; Empatia AND Enfermagem AND Estudantes de Enfermagem; Empatia AND Enfermagem.

Os critérios de inclusão foram: estudos originais (quantitativos, qualitativos, quanti-qualitativos, relato de experiências e estudos de caso), disponíveis na íntegra; publicados em português, inglês ou espanhol, com os resumos disponiveis nas bases de dados selecionadas, publicados em periódicos nacionais e internacionais e que estivessem dentro do recorte temporal de 2009 a 2019.

Os critérios de exclusão foram: pesquisas que investigassem especificamente sobre funcionários de outras áreas da saúde, em ambientes não hospitalares, pesquisas avaliando o grau empático em estudantes de Medicina, que tivessem utilizado escalas aplicadas em estudantes de Medicina ou outra área da saúde e que apresentasse apenas traduções de escalas.

Realizada a análise bibliométrica ${ }^{12-13}$, na sequência foram analisados os achados, através de um quadro sinóptico com os principais resultados. Os artigos foram lidos e relidos para captação de seus conteúdos. Em seguida, foi realizada análise de conteúdo com base no referencial de Minayo ${ }^{14}$, emergindo três categorias temáticas, que são: "Percepção do estudante de Enfermagem acerca do cuidado", "Uma reflexão sobre empatia e a Enfermagem" e "Habilidade empática e suas competências".

Nesta revisão, foi empregado a classificação de evidências nos níveis ${ }^{15}$ : I -oriundas de revisões sistemáticas ou metanálise de relevantes ensaios clínicos; II - pelo menos um ensaio clínico randomizado controlado bem delineado; III - ensaios clínicos bem delineados, sem randomização; IV - estudos de coorte e de caso-controle bem delineados; $V$ - revisão sistemática de estudos descritivos e qualitativos; VI - evidências derivadas de um único estudo descritivo ou qualitativo; e VII 
- opinião de autoridades ou relatório de comitês de especialistas.

\section{Aspectos Éticos}

Apesar de não ser um estudo com seres humanos, os aspectos éticos foram observados e cumpridos em sua execução. Os autores dos artigos analisados foram devidamente referenciados. Os dados e informações das pesquisas foram apresentados de forma fidedigna.

\section{RESULTADOS}

A busca nas bases de dados contabilizou 644 artigos e, destes, 61 foram eleitos para leitura do texto completo e 11 compuseram o estudo, conforme consta no fluxograma da Figura 1.

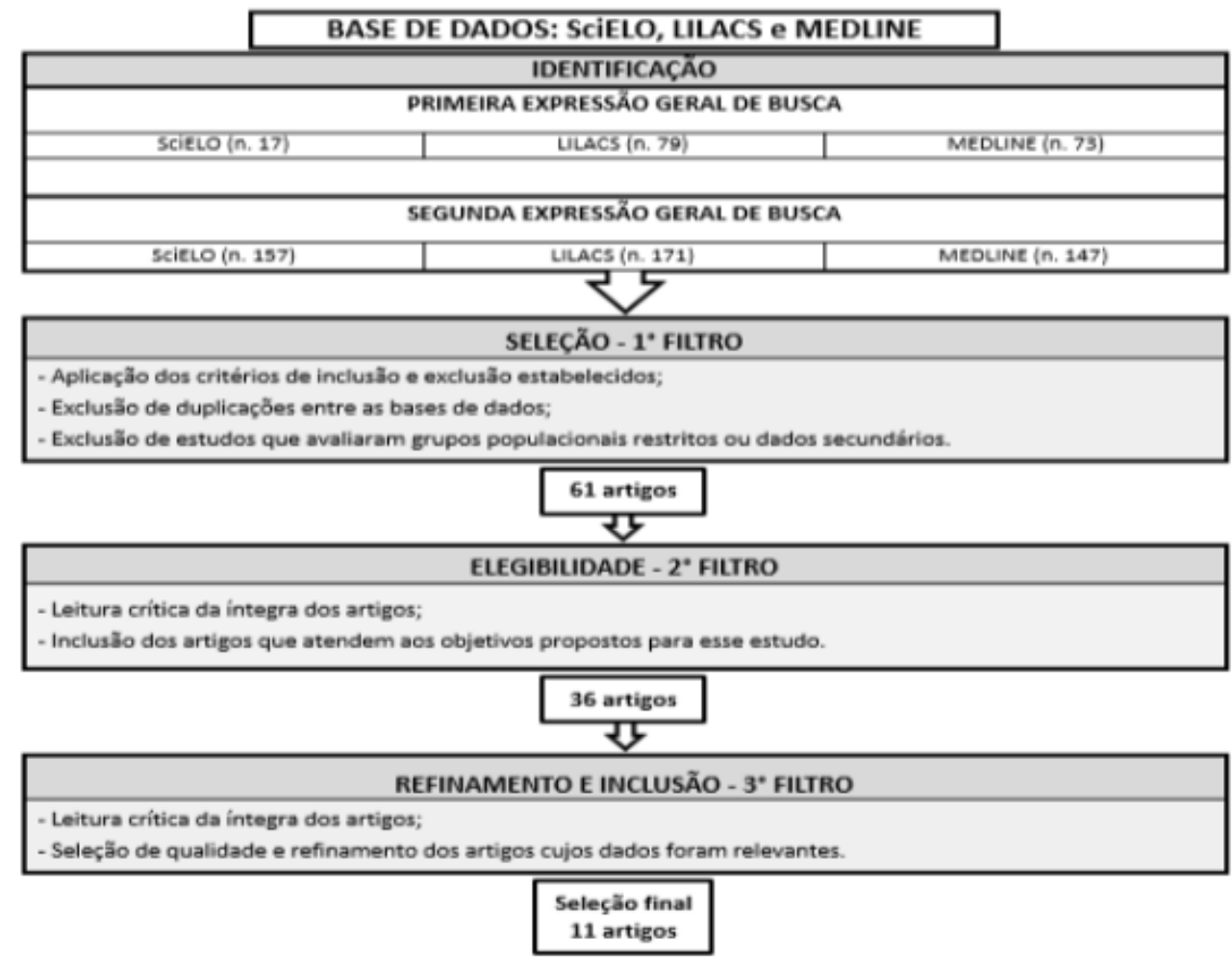

As categorias que emergiram com análise de conteúdo, apontaram o seguinte:

Categoria 1: Percepção do estudante de Enfermagem acerca do cuidado

Nesta categoria trata-se da percepção do estudante de Enfermagem, em que se abordam as definições para o cuidado e como este aparece de acordo com as vivências e experiências. Além disso, a mudança deste conceito conforme o tempo de atuação e estudo, durante o curso. Nesta, foram avaliados cinco artigos, em que os autores concluíram que não há uma definição específica sobre cuidado, mas grande parte destes os correlaciona com a empatia.

Categoria 2: Uma reflexão sobre empatia e a Enfermagem

O maior número de artigos encontrados nesta busca definiu a empatia como habilidade essencial no aprendizado e na prática da Enfermagem. Foram utilizados seis artigos que discutem a importância da empatia no relacionamento entre enfermeiro-cliente. Um artigo foi utilizado para pesquisa sobre a forma mais utilizada para medir o grau de empatia.

As evidências encontradas nessa categoria reforçam a prática do cuidado humanizado e da empatia como competência, e se esta pode ou não ser aprendida, pois se trata de uma habilidade adquirida ao longo da vida e depende da experiência individual de cada estudante.

Categoria 3: Habilidade empática e suas competências.

Respondendo à pergunta levantada na categoria anterior quatro artigos mostraram que a empatia pode ser uma habilidade aprendida, e que este aprendizado é muito benéfico. Apesar de ser uma habilidade aprendida socialmente, é possível exercer uma estratégia para ensinar, por meio de recursos como arte, música e até teatro. Porém para que possa ser ensinada, é necessário que os docentes também possuam um comportamento empático, gerando uma cadeia de empatia que começa no docente, passa pelos graduandos e termina no cliente.

Os artigos incluidos nas categorias, bem como as especificações destes, estão descritos no Quadro 2, resultando na apresentação da revisão/sintese do conhecimento 7 .

Abaixo no Quadro 2 são apresentados de forma resumida os achados deste estudo. 


\begin{tabular}{|c|c|c|c|c|c|c|c|c|c|c|c|}
\hline 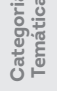 & $\rightarrow$ & $\rightarrow$ & -1 & 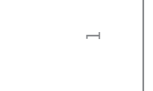 & $\sim$ & N & N & N & $\mathrm{m}$ & $\mathrm{m}$ & m \\
\hline 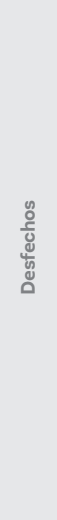 & 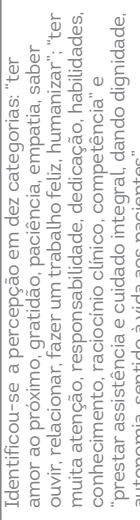 & 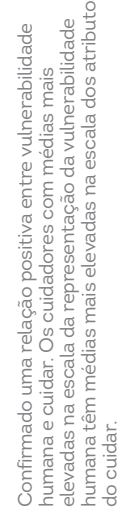 & 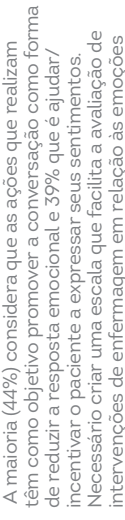 & 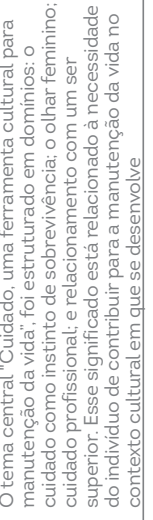 & 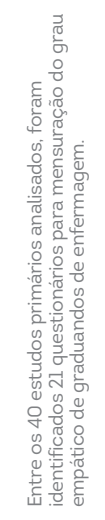 & 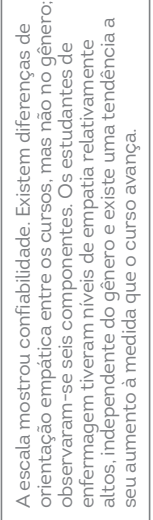 & 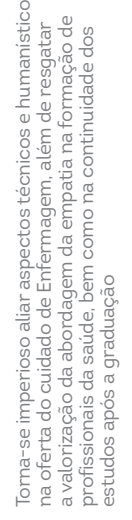 & 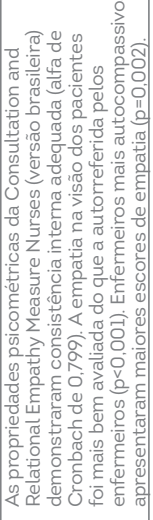 & 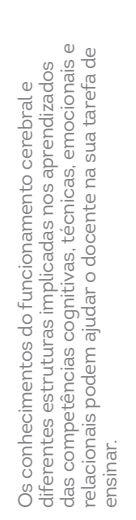 & 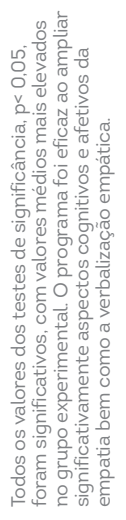 & 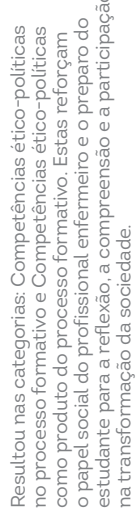 \\
\hline$\frac{\overbrace{\overline{0}}^{\circ}}{\frac{0}{80}}$ & 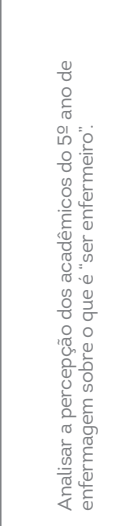 & 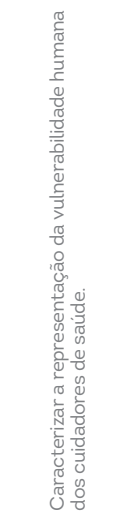 & 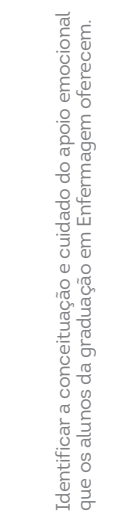 & 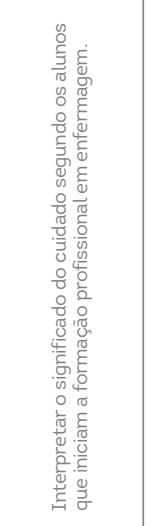 & 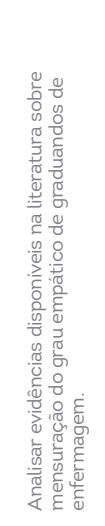 & 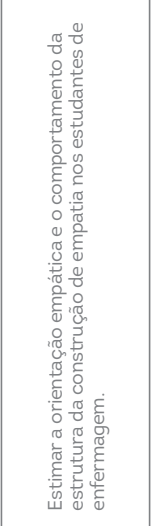 & 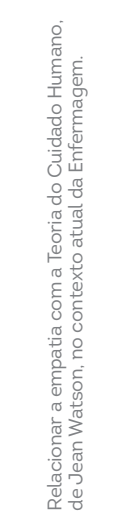 & 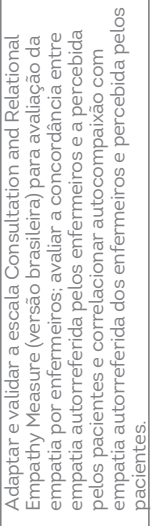 & 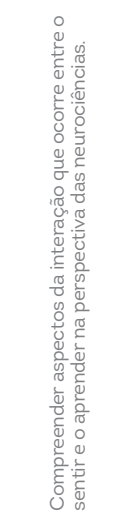 & 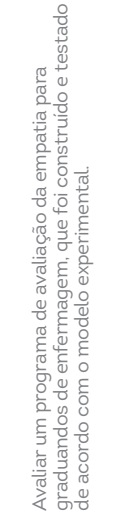 & 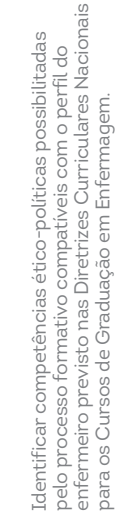 \\
\hline 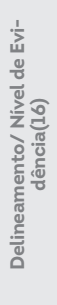 & 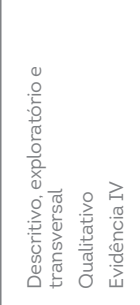 & 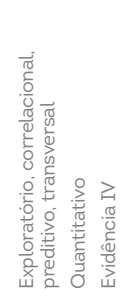 & 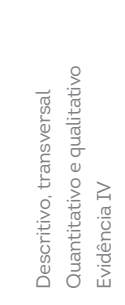 & 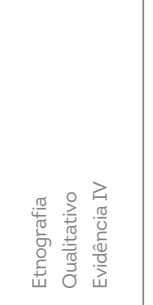 & 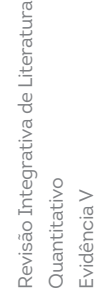 & 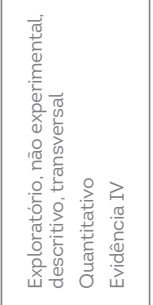 & 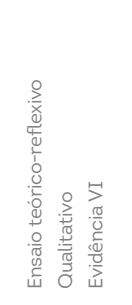 & 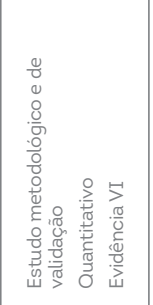 & 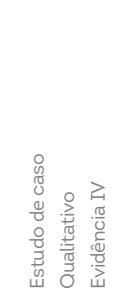 & 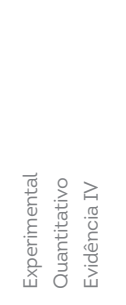 & 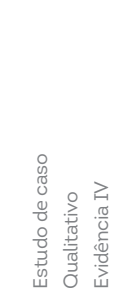 \\
\hline $\begin{array}{l}\frac{\mathscr{\nu}}{2} \\
\frac{5}{4}\end{array}$ & 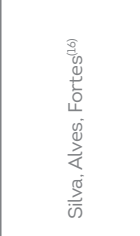 & 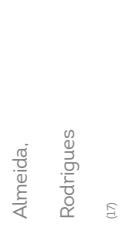 & 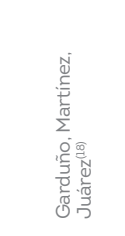 & 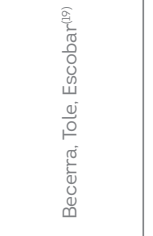 & 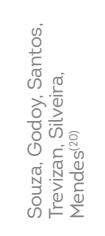 & 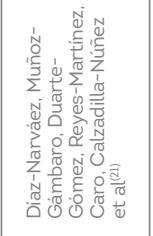 & & & & & 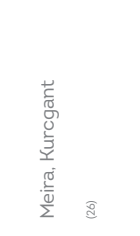 \\
\hline 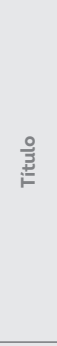 & 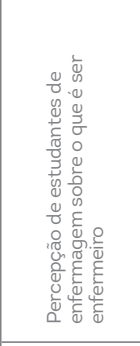 & 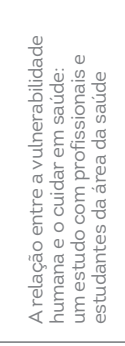 & 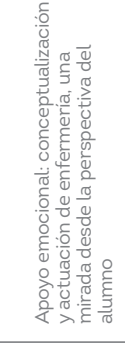 & 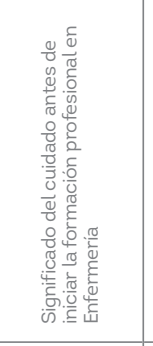 & 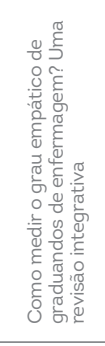 & 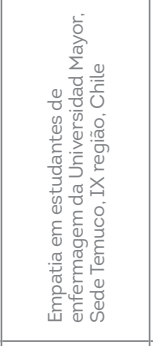 & 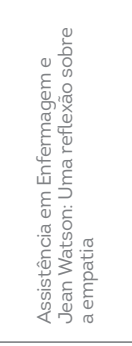 & 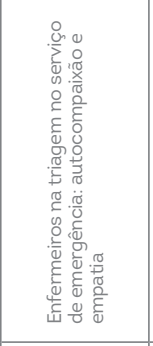 & 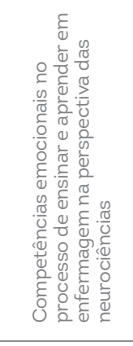 & 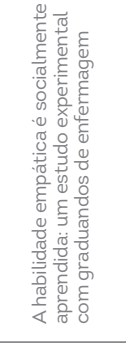 & 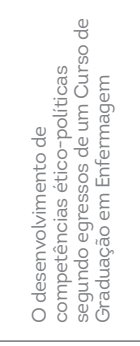 \\
\hline 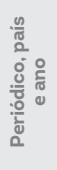 & 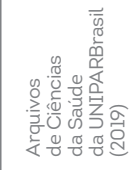 & 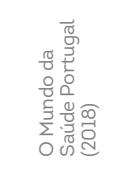 & 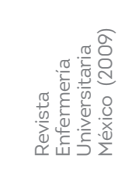 & 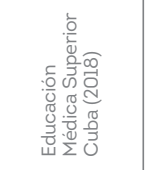 & 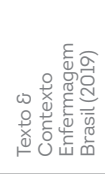 & 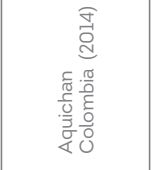 & 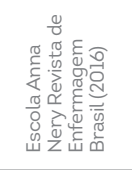 & 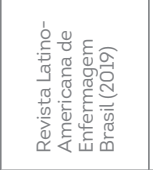 & & 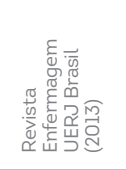 & 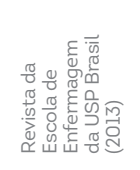 \\
\hline
\end{tabular}




\section{DISCUSSÃO}

Categoria temática 1: Percepção do estudante de Enfermagem acerca do cuidado

A prática da Enfermagem é, muitas vezes, descrita como a arte de cuidar. Este cuidado pode se referir aos aspectos físicos, mentais e sociais. Desta forma, podemos dizer que a Enfermagem tem como missão proporcionar o bem-estar e conforto ao paciente, podendo este ser realizado por meio de uma assistência direta ou coordenando outras atividades ${ }^{16}$

A opção do estudante pelo curso de Enfermagem e conhecimento sobre sua finalidade e a percepção acerca do cuidado está diretamente relacionada à dinâmica social da sociedade a qual estão envolvidos. Assim, este estudante pode ter diferentes visões desde a escolha, até o aprendizado no transcorrer do curso, e atuação prática como enfermeiro ${ }^{16}$.

As Diretrizes Curriculares Nacionais (DCN) do Curso de Enfermagem propõem quais competências e habilidades devem ser estudadas durante a graduação, entendendo que estas são necessárias para a formação de um futuro profissional, que deva agir com afetividade e humanização durante o desenvolvimento do cuidado, sobretudo no Sistema Único de Saúde (SUS) ${ }^{27}$

A entrada em uma universidade é um momento difícil na vida de grande parte dos estudantes, sentimentos de nervosismo, insegurança, estranheza são comuns em situações de adaptação e entrada em um novo grupo social. Os métodos de ensino são distintos, alguns alunos mudam de suas cidades para ingressar em uma universidade em outra região. Assim, ao entrar neste novo mundo de conhecimento, o graduando de enfermagem se desprende das definições que conheceu anteriormente, e se abre para adentrar em um ambiente acadêmico e ressignificar o papel da profissão que exercerá daqui para frente ${ }^{28}$

Alguns estudantes, não sabem exatamente a que se refere o cuidado, como este faz parte da responsabilidade do enfermeiro em exercer um papel empático. Outros, trazem definições próprias do que significa cuidar e tem dificuldades em construir sua identidade como enfermeiro. Estas definições mudam e são aprendidas no decorrer do curso ${ }^{28}$.

Atualmente, existem diversos estudos que trabalham em prol dos cuidados e defesa dos pacientes, para que a qualidade deste cuidado esteja centrada em um cuidado humanizado. O cuidado humanizado tem como percepção, além da valorização do cuidado técnico e científico, também reconhecer a individualidade de cada paciente, sua dignidade, seus direitos, e reconhecendo o profissional como um ser humano ${ }^{17}$.

Durante a formação do enfermeiro, é primordial o aprendizado sobre apoio emocional ao paciente. Porém faltam definições claras sobre este conceito. Para ser capaz de dar este apoio emocional de forma assertiva, o aluno deve possuir conhecimentos científicos dentro das três esferas: biológica, social e psicológica. Assim, o enfermeiro deve ter os conhecimentos necessários e a sensibilidade para atender à necessidade individual dentro da demanda do dia a dia ${ }^{18}$.

Este conceito de cuidado em conjunto com apoio emocional, tem uma natureza complexa, com muitas definições. Algumas das definições dadas por estudantes são "dar confiança, empatia", "prestar cuidados focados nos aspectos físicos e emocionais". A visão humanitária sobre a profissão enfermagem, e sua visão romantizada, associa os profissionais aos sentimentos de paciência e empatia. $E$ isto faz com que o trabalho excessivo esteja relacionado ao contexto de cuidado integral, resultando em constantes manifestações por melhorias nas condições de trabalho, que muitas vezes tornam impossivel, que o profissional pratique o cuidado humanizado ${ }^{19}$

Categoria temática 2: Uma reflexão sobre empatia e Enfermagem

A origem do termo empatia origina-se no final do século XIX, a partir do filósofo alemão Robert Vischer. O termo clássico é empatheia, elaborado pelo filosófico em 1873, a palavra em alemão einfühlung, que traduzida para o português significa "sentir em" ou "sentir-se dentro". Inicialmente ela descrevia a sensação ao observar uma obra de arte, depois passou a descrever sentimentos de conexão com a natureza ${ }^{29}$.

A empatia pode ser descrita como a capacidade de ser sensivel acerca das emoções do outro. Quando é possivel compartilhar sentimentos, e a aptidão de se colocar no lugar de outra pessoa e entender o que está passando. Para os estudantes de enfermagem, a empatia é indispensável na relação com os pacientes. Formando assim, um vínculo que beneficia ambos. Quando o paciente se sente compreendido e acolhido, tende a melhorar a adesão ao tratamento, que, por sua vez, fortalece o sentimento de dever realizado do enfermeiro ${ }^{20}$.

A primeira visão de empatia surge a partir do contato com os pais, esta atitude por parte dos primeiros modelos de vida, é fundamental para que os filhos se tornem adultos saudáveis, e lhes confere a habilidade empática com outras pessoas, assim como seus pais foram anteriormente. Para ter uma relação empática com seus pacientes, é preciso que profissionais da saúde estejam em contato com os próprios sentimentos de forma que consigam entender os sentimentos dos outros, e os diferenciar dos seus. Dessa forma, o profissional consegue se colocar no lugar do próximo, estabelecendo uma relação empática ${ }^{30}$

Uma das ferramentas mais utilizadas para medir o grau de 
empatia, é a Escala de Empatia Médica de Jefferson (EEMJ), sua estrutura é multidimensional e tem três fatores principais: a perspectiva, atenção com compaixão, e a habilidade de "se colocar nos sapatos" dos pacientes. Há diversos estudos sobre empatia com estudantes da área da saúde, porém são, em sua maioria, sobre estudantes de medicina ${ }^{21}$.

Estas questões sobre o atendimento de saúde de forma humanizada e empática, tem ganhada destaque por parte dos profissionais. Países tem investido em estudos que incentivem os médicos e enfermeiros a se comportarem de maneiros mais pessoal durante o atendimento, para que este seja estendido as demais questões humanas. Segundo a Teoria do Cuidado Humano, um dos elementos mais importantes na construção da relação de confiança entre profissional da saúde-paciente é a empatia ${ }^{22}$

Sendo assim, a empatia surge como uma tática para reforçar as demandas por este cuidado humanizado. A empatia e a compaixão são características essenciais para o processo de aprendizado da profissão enfermagem. Sendo imprescindiveis para o estudante, porém esta é uma habilidade que pode ser ensinada e, consequentemente, aprendida ${ }^{23}$.

Categoria temática 3: Habilidade empática e suas competências

Para o docente, é necessário que se contorne as dificuldades e desenvolva estratégias de ensino que abranjam os nossos conhecimentos da neurociência, para otimizar a aprendizagem do aluno. Há de se pensar o ensinar como uma estratégia que valoriza o sensivel e o emocional, em detrimento de ser apenas executado, com uma prática repetitiva, e mecânica ${ }^{24}$

A empatia é uma habilidade aprendida socialmente, carecendo de capacitação profissional que desenvolvam um comportamento empático, e focando em uma escuta ativa e acolhedora. É na infância que se aprende habilidades sociais como a empatia, ainda que surjam oportunidades para desenvolvê-la ao longo da vida. Locais que incluem a empatia com uma aprendizagem comum e formal, tem mostrado resultados benéficos ${ }^{25}$

A aprendizagem de competências socioeducativas e ético-políticas é um dos desafios para a formação de enfermeiros. A ação humana tem caráter político, pois sempre integra interesses pessoais e coletivos. No entanto, o que deve determinar a ação e decisões de profissionais da saúde são os valores e principios éticos ${ }^{26}$

Também é um desafio para os docentes e educadores reinventarem o sistema de ensino da enfermagem para que este não seja apenas tratado como ciência biológica, ainda que o aprendizado de técnicas seja muito importante. É um sistema engessado, que precisa de um novo olhar para o cuidado humanizado e a importância das relações humanas. Estas, que podem ser tão importantes quando o cuidado sistematizado. Cabe aos educadores ouvirem os alunos, abrindo uma reflexão e os motivando ${ }^{31}$

A habilidade de ouvir ativamente o outro, sem julgamento, é a principal compreensão para o aprendizado da empatia. Esta capacidade deve estar em conjunto com a habilidade de identificar os próprios sentimentos, de forma que permita se colocar no lugar do outro. Se o profissional não consegue distinguir e regular suas próprias emoções, foca a atenção em si mesmo, fazendo com que torne difícil atingir o comportamento de ajuda e o compreendimento da necessidade do paciente, o que pode prejudicar o cuidado ${ }^{25}$.

Alguns estudos mostram que a literatura, poesia e cinema, bem como outras artes criativas, em conjunto com discussões em que se tem por objetivo entender o ponto de visto do paciente, tem resultados muito positivos para o ensino da empatia. Outras estratégias como treinamento das habilidades de comunicação, intervenções teatrais (onde o graduando se coloca no lugar do paciente) também resultaram no aumento dos níveis de empatia ${ }^{29}$.

\section{Limitações do estudo}

Uma importante limitação deste estudo é o baixo nível de evidência dos artigos. Outro aspecto é que grande parte dos estudos são focados em estudantes de Medicina, e poucos na Enfermagem.

\section{Contribuições para a prática}

O estudo contribuiu para a análise da temática no ensino de Enfermagem, possibilitando a inclusão da temática na grade curricular, bem como evidenciou a importância do cuidado humanizado em todas as áreas da saúde, para construção e um relacionamento com o paciente que é benéfica para todos os envolvidos.

Sendo assim, o estudante de enfermagem deve ser instruído acerca da empatia e relacionamento com o paciente, bem como estar apto a realizar um atendimento humanizado. Este estudo poderá ser utilizado em estudos futuros para a estruturação do ensino da empatia na grade curricular das universidades, e como isso pode ser melhorado e intensificado para uma melhor prática na relação paciente-enfermeiro.

\section{CONCLUSÃO}

Os resultados obtidos a partir desta revisão integrativa na literatura, possibilitaram que empatia fosse vista como capacidade essencial na formação de estudantes de enfermagem. Esta habilidade está diretamente relacionada ao cuidado humanizado e mais pessoal por parte da equipe de saúde. 
O graduando de Enfermagem, ao ingressar na universidade, está em conflito com seus sentimentos, devido a mudança de ambiente e para que haja a prática da empatia, é preciso que o profissional da saúde esteja em contato com os seus sentimentos primeiro. Além disso, o estudante entra com a sua própria visão do significado do cuidado e da habilidade empática, de acordo com os seus aprendizados, experiências e situações vivenciadas durante a vida, podendo ser uma visão romantizada ou uma habilidade que não foi aprendida.

A empatia como habilidade pode ser ensinada desde que haja uma ligação entre o docente e o aluno, pois para ensinar uma habilidade como esta, é preciso que o docente reveja seus métodos de ensino de forma menos impessoal também. Portanto, esta deve fazer parte da grade de ensino em todas as áreas da saúde. Todos são beneficiados com o aprendizado desta capacidade, pois fortalece o vínculo paciente-enfermeiro, e caracteriza o cuidado como não apenas mecânico e científico, mas humano.

\section{Contribuição dos Autores:}

LS Rondon e ICKO Cunha participaram da concepção e delineamento do estudo, redação e revisão do conteúdo intelectual até a versão final do manuscrito. FRG Ximenes Neto participou do delineamento, da redação e revisão do conteúdo intelectual até a versão final do manuscrito.

Financiamento: Bolsa de Iniciação Científica PIBIC/ CNPQ 2019-2020 para a segunda autora.

\section{REFERÊNCIAS}

1. Angerami ELS. O mister da investigação do enfermeiro. Rev. Latino-Am. Enfermagem [Internet]. 1993 [citado 2020 Mar 30];1(1):11-22. Available from:: http://www.scielo.br/scielo.php?script=sci_arttextEpid=SO104116919930001000038 lng=en.

2. Brasil. Lei 7498/86. Dispõe sobre a regulamentação do exercício da Enfermagem e dá outras providências. Available from: http://www.cofen.gov.br/lei-n-749886-de-25-de-junho-de-1986_4161.html.

3. Takaki MH, Sant'Ana DMG. A empatia como essência do cuidado prestado ao cliente pela equipe de enfermagem de uma unidade básica de saúde. Cogitare Enferm [Internet] 2004 [citado 2020 Mar 30];9(1):79-83. Available from: https://revistas.ufpr.br/cogitare/article/view/1708.

4. Falcone E. A avaliação de um programa de treinamento da empatia com universitários. RBTCC [Internet]. 1999 [citado 2020 Mar 30];1(1):23-2. Available from: http://pepsic.bvsalud.org/scielo.php?script=sci_arttextEpid=S151755451999000100003 ing=ptEnrm=iso.

5. Nascimento HCF, Ferreira Júnior WA, Silva AMTC, Carvalho IGM, Bastos GCFC, Almeida RJ. Análise dos niveis de empatia de estudantes de medicina. Rev. bras. educ. méd. [Internet]. 2018 [citado 2020 Mar 30];42(1):150-8. Available from: http://www.scielo.br/scielo.php?script=sci_arttextEpid=SO100-550220180001001528lng=en\&nr m=iso.

6. Conselho Nacional de Educação. Câmara de Educação Superior. Resolução CNE/CES 3/2001. Diário Oficial da União, Brasilia, 9 de Novembro de 2001. Seção 1, p. 37. Available from: http://portal.mec.gov.br/cne/arquivos/pdf/ CESO3.pdf

7. Instituto Ayrton Senna [Internet]. Descubra como competências socioemocionais podem melhorar a Educação brasileira. 2019 [Acesso em 2020 Abr 11]. Available from:: https://institutoayrtonsenna.org.br/pt-br/meueducador-meu-idolo/materialdeeducacao/descubra-como-competencias-socioemocionais-podem-melhorara-educacao-brasileira.html.

8. SouzaMT,SilvaMD, CarvalhoR.Integrativereview: whatisit?Howto doit?[Internet].2010[cited 2020 Mar30];8(1):102106. Available from: http://www.scielo.br/scielo.php?script=sci_arttextEpid=S1679-450820100001001028lng=en.

9. Mendes KDS, Silveira RCCP, Galvão CM. Revisão integrativa: método de pesquisa para a incorporação de evidências na saúde e na enfermagem. Texto contexto - enferm. [Internet]. 2008 [citado 2020 Mar 30];17(4):758-764. Available from:http://www.scielo.br/scielo.php?script=sci_arttextEpid=S0104-07072008000400018Elng=en.

10. Santos CMC, Pimenta CAM, Nobre MRC. The PICO strategy for the research question construction and evidence search. Rev. Latino-Am. Enfermagem [Internet]. 2007 [cited 2020 Mar 30];15(3):508-51l. Available from: http:// www.scielo.br/scielo.php?script=sci_arttext\&pid=S0104-11692007000300023\&lng=en.

11. Esteves LSF, Cunha ICKO, Bohomol E, Negri EC. Supervised internship in undergraduate education in nursing: integrative review. Rev. Bras. Enferm. [Internet]. 2018 [cited 2020 Apr 10];71(Suppl 4):1740-1750. Available from: http://www.scielo.br/scielo.php?script=sci_arttext\&pid=S0034-71672018001001740\&lng=en.

12. Soares CB, Hoga LAK, Peduzzi M, Sangaleti C, Yonekura T, Silva DRAD. Integrative Review: Concepts And Methods Used In Nursing. Rev. esc. enferm. USP [Internet]. 2014 [cited 2020 Mar 30];48(2):335-345. Available from: http:// www.scielo.br/scielo.php?script=sci_arttext\&pid=S0080-62342014000200335\&lng=en. 
13. Neves VR, Sanna MC. Nursing leadership teaching: a bibliometrics study. Acta paul. enferm. [Internet]. 2012 [cited 2020 Mar 30];25(2):308-313. Available from: http://www.scielo.br/scielo.php?script=sci_arttextEpid=SO103210020120002000248 lng=en.

14. Minayo MCS. (Org.). Pesquisa social: teoria, método e criatividade. 26 ed. São Paulo: Editora Vozes; 2007.

15. Pereira AL, Bachion MM. Atualidades em revisão sistemática de literatura, critérios de força e grau de recomendação de evidência. Rev. Gaúcha Enferm. [Internet]. 2006 [citado 2020 Abr 10];27(4):491. Available from:https://seer.ufrgs. br/RevistaGauchadeEnfermagem/article/view/4633/2548.

16. Silva BLG, Alves ES, Fortes AFA. Percepção de estudantes de enfermagem sobre o que é ser enfermeiro. Arq. Cienc. Saúde UNIPAR. [Internet]. 2019 [citado 2020 Abr 07];23(2):81-88. Available from: https://www.revistas.unipar.br/ index.php/saude/article/view/6136/3771.

17. Almeida CMT, Rodrigues VM. The relationship between human vulnerability and health care: a study with professionals and students in the health area. Mundo saúde (Impr.). 2018 [cited 2020 Apr 07];42(4):992-1011. Available from: https://www.revistamundodasaude.com.br/uploads/20180148.PDF.

18. Garduño MDM, Martínez VG, Juárez PS. Apoyo emocional: conceptualización y actuación de enfermería, una mirada desde la perspectiva del alumno. Enfermería Universitaria. 2009 [citado 2020 Abr 07];6(3):35-39. Available from: https://www.medigraphic.com/pdfs/enfuni/eu-2009/eu093f.pdf.

19. Becerra AC, Tole MG, Escobar LMV. Significado del cuidado antes de iniciar la formación profesional en Enfermería. Educación Médica Superior [Internet]. 2018 [citado 2020 Abr 07];32(3):[aprox. 0 p.]. Available from: http://www.ems. sld.cu/index.php/ems/article/view/1471.

20. Souza MC, Godoy S, Santos SS, Trevizan MA, Silveira RCCP, Mendes IAC. How to measure the empathy level of undergraduate nursing students? an integrative review. Texto contexto - enferm. [Internet]. 2019 [cited 2020 Apr 07];28:e20180017. Available from: http://www.scielo.br/scielo.php?script=sci_ arttext\&pid=S0104-070720190001005098lng=en.

21. Díaz-Narváez VP, Muñoz-Gámbaro G, Duarte-Gómez N, Reyes-Martínez MC, Caro SE, Calzadilla-Núñez A et al. Empatia en estudiantes de enfermería de la Universidad Mayor, sede Temuco, IX región, Chile. Aquichan [Internet] 2014 [cited 2020 Apr 07];14(3):388-402. Available from: http://www.scielo.org.co/scielo.php?script=sci_ arttext\&pid=S1657-59972014000300009\&lng=en.

22. Savieto RM, Leão ER. Assistência em Enfermagem e Jean Watson: Uma reflexão sobre a empatia. Esc. Anna Nery [Internet]. 2016 [cited 2020 Apr 07];20(1):198-202. Available from: http://www.scielo.br/scielo.php?script=sci_ arttext\&pid=S1414-.81452016000100198\&lng=en

23. Savieto RM, Mercer S, Matos CCP, Leão ER. Enfermeiros na triagem no serviço de emergência: autocompaixão e empatia. Rev. Latino-Am. Enfermagem [Internet]. 2019 [cited 2020 Apr 07];27:e3151. Available from: http://www. scielo.br/scielo.php?script=sci_arttextEpid=S0104-11692019000100340\&lng=en.

24. Pereira WR, Ribeiro MRR, Depes VBS, Santos NC. Emotional competencies in the process of teaching and learning in nursing, from the perspective of the neurosciences. Rev. Latino-Am. Enfermagem [Internet]. 2013 [cited 2020 Apr 07];21(3):663-669. Available from: http://www.scielo.br/scielo.php?script=sci_arttextEpid=SO104$11692013000300663 \&$ lng=en.

25. Kestenberg CCF. The empathic skill is socially learned: an experimental study with nursing undergraduate students. Revista Enfermagem UERJ. 2014 [cited 2020 Apr 07];21(4):427-433. Available from: https://www.e-publicacoes.uerj. br/index.php/enfermagemuerj/article/view/8892.

26. Meira MDD, Kurcgant P. Political-ethical skill development in nursing undergraduates. Rev. esc. enferm. USP[Internet]. 2013 [cited 2020 Apr 07];47(5):1203-1210. Availablefrom: http://www.scielo.br/scielo.php?script=sci_ arttextEpid=S0080-62342013000501203\&lng=en.

27. Silva ANC, Moreira DP, Freitas CMA, Teixeira AKS, Pinheiro ARM. Estágio extracurricular de Enfermagem: estratégia para a formação profissional. Enferm. Foco, [S.L.]. 2019. [citado 2020 Abr 12];10(4):129-135. Available from: http:// revista.cofen.gov.br/index.php/enfermagem/article/view/1880.

28. Queiroz BFB, Garanhani ML. Construindo significados do cuidado de enfermagem no processo de formação: uma pesquisa fenomenológica. Cienc. Cuid. Saúde [Internet]. 2012 [citado 2020 Abr 11];11(4):775-783. Available from: http://periodicos.uem.br/ojs/index.php/CiencCuidSaude/article/view/21660.

29. Schweller M. O ensino de empatia no curso de graduação em medicina. Tese (doutorado) - Universidade Estadual de Campinas, Faculdade de Ciências Médicas. 2014 [citado 2020 Abr 10];137 p. Available from: http://www.repositorio. unicamp.br/handle/REPOSIP/313594

30. Terezam R, Reis-Queiroz J, Hoga LAK. The importance of empathy in health and nursing care. Rev. Bras. Enferm. [Internet]. 2017 [cited 2020 Apr 10];70(3):669-670. Available from: http://www.scielo.br/scielo.php?script=sci_ arttextEpid=S0034-716720170003006698lng=en 